\title{
EKF Based GPS/Odometer Data Fusion For Precise Robot Localization In Outdoor Environment
}

\author{
Chinmaya Kumar Mishra ${ }^{1}$, B.K. Mishra ${ }^{2}$, J. Sam Jebakumar ${ }^{3}$ \\ ${ }^{1,3}$ SRM University, Kattankulathur.Chennai \\ ${ }^{2}$ BARC, Mumbai
}

\begin{abstract}
This paper describes a method of data fusion for outdoor autonomous robot equipped with onboard GPS receiver and Odometer using Extended Kaman filter. Both GPS and Odometer having their own advantage and disadvantage for accurate position information of Robot in unknown/outdoor environment. Described Data fusion technique fuse position input of both Odometer and GPS to generate most accurate position estimation with less uncertainty (standard deviation) by using Extended Kalman Filter. Once precise position of Robot obtained in unknown environment then other control actions can be applied on Robot to put it on the right track/Path.
\end{abstract}

Simulation of fusion technique and all results has been done in MATLAB with proper units and values.

Keywords:EKF, Odometer, Inertial Sensors, GPS, Data Fusion, Localization.

\section{INTRODUCTION}

Robot navigation in indoor environment is easy due to availability of multiple precise sensor as well as well known environment/surrounding. But in outdoor navigation unknown surrounding as well as limited number of precise positioning sensors are main constrain for navigation. Generally Inertial measurement unit (IMU)/Odometer with GPS sensors are most used positioning sensors in outdoor navigation. Both GPS and Odometer having their own advantage and disadvantage for accurate position information of Robot in unknown environment. Here mainly Odometer and GPS sensor has been used for analysis and simulation. Brief description, advantage and limitation of both Sensors are listed as below,

1) GLOBAL POSITIONING SYSTEM :

The GPS System was launched in 1993 and become fully operational in 1995. According to information provided by the US department of defence, GPS receivers can achieve a position measurement accuracy of 3 meters horizontally and 5 meters vertically,95\% of the time. However this is highly dependent on the surrounding of the receiver. With a constellation of 24 operational satellites working in six different orbits, four satellites on each orbit, the GPS system can achieve coverage all over the world, which implies visibility of between four to ten satellites continuously to have the GPS system working, accurate time stamps have to be Embedded together with information about the satellite position and this is made possible by four atomic clocks available on each satellite.

i) Advantages:

- Works with accuracy, irrespective to environment and movement of system.

- Can be used to obtain estimation of position as well as velocity.

- Useful in Robot localization in unknown and hazardous environment.

ii) Limitations:

- Satellite error and relativistic effect due to satellite orbit deviation and small time error in atomic clock of satellite.

- GPS signal can travel at the speed of light only in vacuum,hence once it inters in atmosphere gets effected and time delay introduced in transmitted signal.

- Measurements get effected with geographical condition, as GPS dose not works in tunnels or inside a building.

- Accuracy is highly effected due to Multipath errors, receiver errors and noise etc.

\section{2) ODOMETER :}

A wheel odometer, sometime referred to as anodograph, is an instrument that measures the distance travelled by counting the number of revolution. Generally with wheel odometery we can obtain rotation information of individual wheel of vehicle and ultimately the distance travelled by individual wheel of vehicle .

i) Advantage:

- Highly accurate measurement for initial movement. 
- Cannot subjected to external disturbances.

- No interference with other sensor signals

\section{ii) Limitation:}

- The position estimate diverges over time, i.e., error increases over time.

- Miscalculations can be caused by slippage and the odometer accuracy varies depending on environment such as temperature and air pressure in the tires.

- High maintenance cost and longer calibration time.

The advantage of both sensors can be utilized with fewer limitations by Extended Kalman Filter based data fusion for most precise localization. As system error is highly nonlinear, here Extended Kalman Filter with Taylor series expansion based linearization has been used. A basic block representation of fusion algorithm and proposed output is as below;

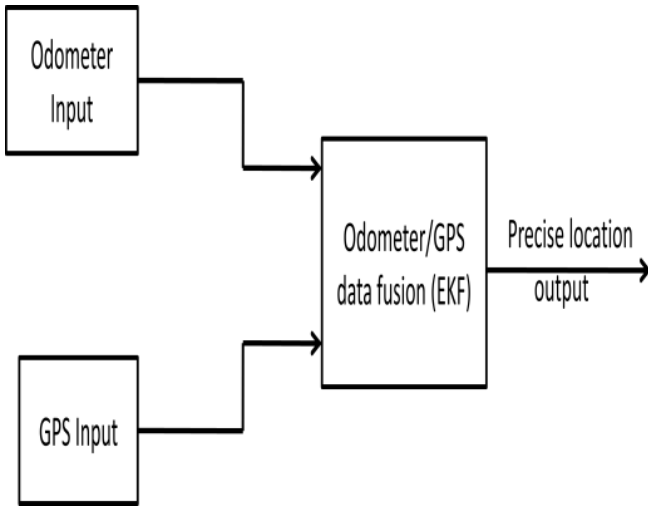

Fig1, Block Representation of Data Fusion

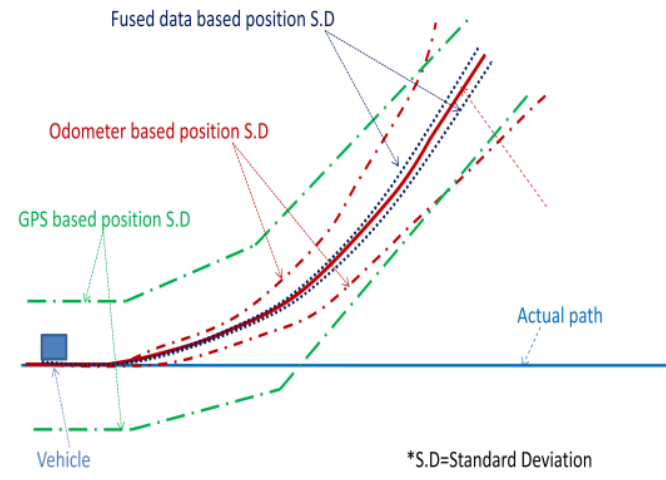

Fig2, Representation of Overall Position estimation improvement by Data Fusion.

In this paper section-Iis used to odometer based robot movement error modelling and GPS based position estimate generation for simulation purpose. Section-II describes Extended Kaman Filter based data fusion technique. Section-III is used for simulation results and analysis.

\section{SECTION-I}

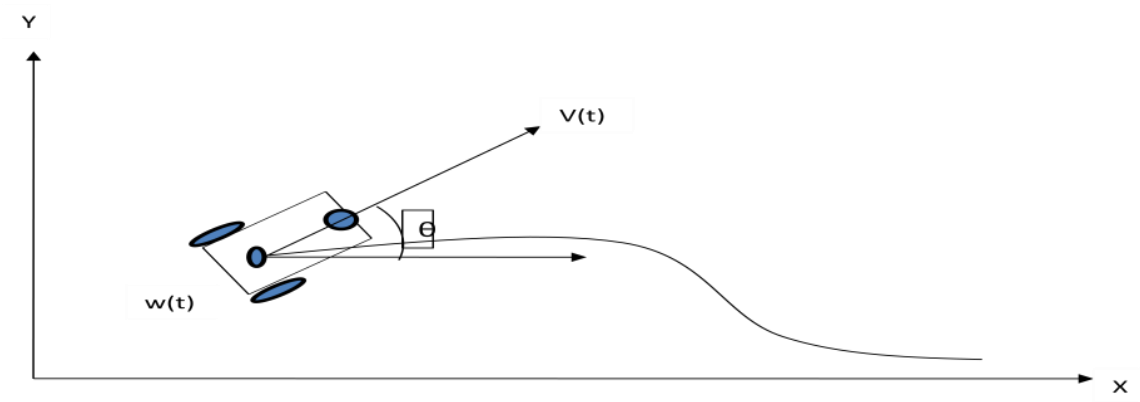

Fig 14, Differential robot with odometer on board

In odometry (wheel sensor only) and dead reckoning (also heading sensor) the position update is based on proprioceptive sensors. The movement of robot sensed with wheel encoders or heading sensors or both is integrated to compute position. Because the sensor measurement errors are integrated the position error accumulates over time. Thus the position has to be updated from time to time by other localization mechanisms; otherwise the robot is not able to maintain a meaningful position estimate in the long run. GPS based position will be around the odometer position with some fixed standard deviation (In this paper it has assumed 2 meters). The mathematical modelling result for odometer and GPS based position estimate as well as nonlinearities are listed as below: 
1) Odometer based position mathematical model result:

$$
\begin{aligned}
& P=\left[\begin{array}{l}
x \\
y \\
\theta
\end{array}\right] \\
& P^{\prime}=f\left(x, y, \theta, \Delta d_{r}, \Delta d_{l}\right)=\left[\begin{array}{l}
x \\
y \\
\theta
\end{array}\right]+\left[\begin{array}{c}
\frac{\Delta d_{r}+\Delta d_{l}}{2} \cos \left(\theta+\frac{\Delta d_{r}-\Delta d_{l}}{b}\right) \\
\frac{\Delta d_{r}+\Delta d_{l}}{2} \sin \left(\theta+\frac{\Delta d_{r}-\Delta d_{l}}{b}\right) \\
\frac{\Delta d_{r}-\Delta d_{l}}{b}
\end{array}\right]
\end{aligned}
$$

Where

$(\mathrm{P}$ and $\mathrm{P} ')=$ Initial and updated position of Robot

$(\Delta \boldsymbol{x} ; \Delta \boldsymbol{y} ; \Delta \boldsymbol{\theta})=$ Path traveled in the last sampling interval.

$\Delta \boldsymbol{d}_{r} ; \Delta \boldsymbol{d}_{\boldsymbol{l}}=$ Distance travelled by right and left wheel respectively.

$\mathrm{b}=$ Distance between the two wheel of the differential-drive robot.

\section{2) GPS based position for simulation purpose:}

GPS position for simulation has been obtained by adding 2 sigma Gaussian noise in Odometer position as it has assumed almost $95 \%$ value will fall with this range. Value of sigma can be changed according to GPS availability and signal strength for a particular situation. The mathematical formula for a generalised GPS data generation for simulation can be written as below;

GPS based position data $=$ Odometer based position data $+\mathbf{n} \times$ Sigma Gaussian noise.

3) Odometer based position covariance and Jacobian mathematical model to estimate nonlinearity:

- Jacobian due to change in position:

$$
F_{p}=\Delta_{p} f=\Delta_{p} f^{T}=\left[\begin{array}{lll}
\frac{\partial f}{\partial x} & \frac{\partial f}{\partial y} & \frac{\partial f}{\partial \theta}
\end{array}\right]=\left[\begin{array}{ccc}
1 & 0 & -\Delta d \sin \left(\theta+\frac{\Delta \theta}{2}\right) \\
0 & 1 & \Delta d \cos \left(\theta+\frac{\Delta \theta}{2}\right) \\
0 & 0 & 1
\end{array}\right]
$$

- Initial Robot Jacobian due to slippage and $\mathrm{d}_{\mathrm{r}}$ and $\mathrm{d}_{\mathrm{l}}$ :

$$
\begin{aligned}
& \sum_{\Delta}=\operatorname{covar}\left(\Delta d_{r}, \Delta d_{l}\right)=\left[\begin{array}{cc}
s_{r}\left|\Delta d_{r}\right| & 0 \\
0 & s_{l}\left|\Delta d_{l}\right|
\end{array}\right] \\
& F_{\Delta r l}=\left[\begin{array}{cc}
\frac{1}{2} \cos \left(\theta+\frac{\Delta \theta}{2}\right)-\frac{\Delta d}{2 b} \sin \left(\theta+\frac{\Delta \theta}{2}\right) & \frac{1}{2} \cos \left(\theta+\frac{\Delta \theta}{2}\right)+\frac{\Delta d}{2 b} \sin \left(\theta+\frac{\Delta \theta}{2}\right) \\
\frac{1}{2} \sin \left(\theta+\frac{\Delta \theta}{2}\right)+\frac{\Delta d}{2 b} \cos \left(\theta+\frac{\Delta \theta}{2}\right) & \frac{1}{2} \sin \left(\theta+\frac{\Delta \theta}{2}\right)-\frac{\Delta d}{2 b} \cos \left(\theta+\frac{\Delta \theta}{2}\right) \\
\frac{1}{b} & -\frac{1}{b}
\end{array}\right]
\end{aligned}
$$

Here assumed that at the starting point the initial covariance matrix $\sum_{p}$ is known.

$\left(\boldsymbol{s}_{\boldsymbol{r}} ; \boldsymbol{s}_{l}\right)=$ Error constant representing the nondeterministic parameters of motor drive and wheel floor interaction. These two constants depend on robot and environment.

Generally the error in position is non-linear in nature. If we assume that $\mathrm{P}$ and $\Delta \boldsymbol{r l}=\left(\Delta \boldsymbol{d}_{\boldsymbol{r}}, \Delta \boldsymbol{d}_{\boldsymbol{l}}\right)$ are uncorrelated, the derivation of $\mathrm{f}\left(\mathrm{p}^{\prime}\right)$ (from equation- ii) can be approximated by the first order Taylors expansion for linearization. Using the error propagation low,

$\sum_{P^{\prime}}=\Delta_{p} f \sum_{p} \cdot \Delta_{p} f^{T}+\Delta_{\Delta r l} f \sum_{\Delta} \cdot \Delta_{\Delta r l} f^{T}$

The covariance matrix $\sum_{p}$. is, of course always given by the $\sum_{p}$. of the previous step and can be calculated after specifying an initial value (e.g.,0).

\section{SECTION-II}

The stage and step of Extended Kaman Filter can be illustrated by below block diagram; 


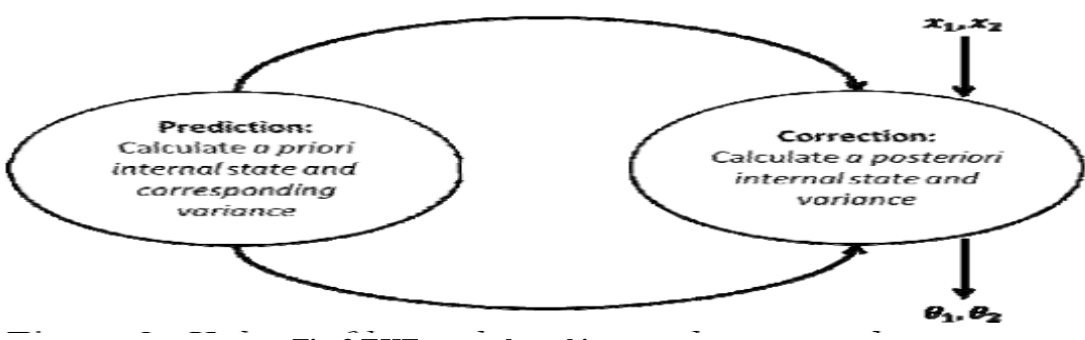

Fig-3,EKF general working sequence.

The data fusion technique based on EKF uses the position and nonlinearity related data from both Odometer as well as GPS and generates a new precise data with improved covariance value. The main function of this system is to reduce the standard deviation in new position compare to given sensor inputs. The mathematical expression for EKF based data fusion is written as below,

$\widehat{\mathrm{q}}=\mathrm{q}_{1}+\frac{\sigma^{2}{ }_{1}}{\sigma^{2}{ }^{1}+\sigma^{2}{ }_{2}}\left(\mathrm{q}_{2}-\mathrm{q}_{1}\right)$

$\sigma_{\mathrm{k}+1}^{2}=\sigma_{\mathrm{k}}^{2}-\mathrm{K}_{\mathrm{k}+1} \sigma_{\mathrm{k}}^{2}$

Where,

$\widehat{\mathrm{q}}=$ New updated position after data fusion.

$\mathrm{q}_{1}=$ Odometer based data of position.

$\mathrm{q}_{2}=$ GPS based data of position.

$\sigma^{2}{ }_{1}=$ Variance in Odometer based position .

$\sigma_{2}^{2}=$ Variance in GPS based position .

$\sigma_{\mathrm{k}+1}^{2}=$ Updated variance in new position

$\mathrm{K}_{\mathrm{k}+1}=\frac{\sigma^{2}{ }_{\mathrm{k}}}{\sigma^{2}{ }_{\mathrm{k}}+\sigma^{2}{ }_{\mathrm{z}}} ; \sigma^{2}{ }_{\mathrm{k}}=\sigma^{2}{ }_{1}$ and ${\sigma^{2}}_{\mathrm{z}}=\sigma^{2}{ }_{2}$

The new ,fused estimation of robot position provided by Extended Kalman Filter is again subject to a Gaussian probability density curve. Its mean and variance are simply functions of the input means and variances. Thus the Extended Kalman Filter provides both a compact, simplified representation of uncertainty and an extremely efficient technique for combining heterogeneous estimates to yield a new estimate for our Robots position.

\section{SECTION-III}

Initial assumptions and values has been taken as below for simulation;

$\mathrm{dr}=0.025$

$\mathrm{dl}=0.010$

$\mathrm{Sr}=0.0025$

$\mathrm{Sl}=0.0010$

GPS Standard deviation $=2$ meters.

No of point simulated=200, All dimensions ( $\mathrm{X}$-axis and $\mathrm{Y}$-axis) are in meter

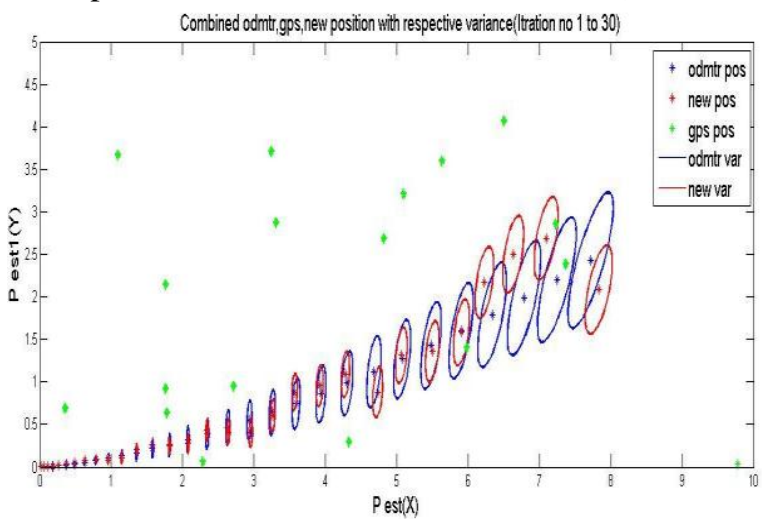

Fig-4, Improved new estimated position withvariance with respect to Odometer position with variance

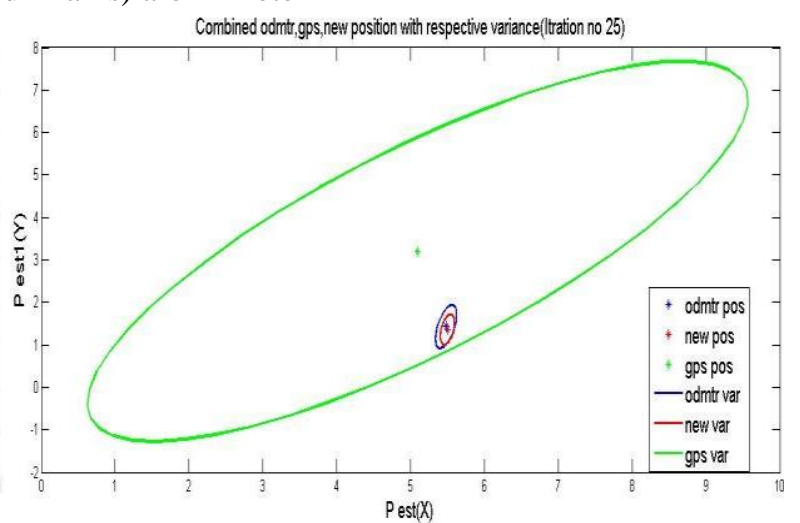

Fig-5, Improved new estimated position variance with respect to Odometer and GPS position and variance. 


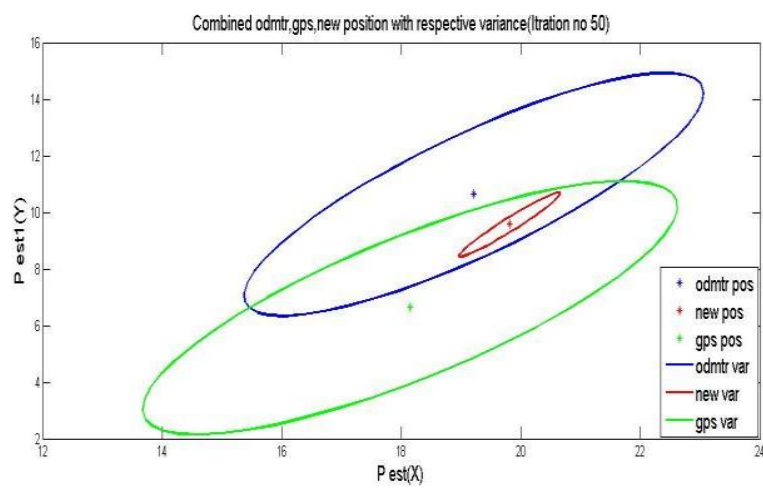

Fig-6,Itration where GPS and Odometer variance becomes almost same.

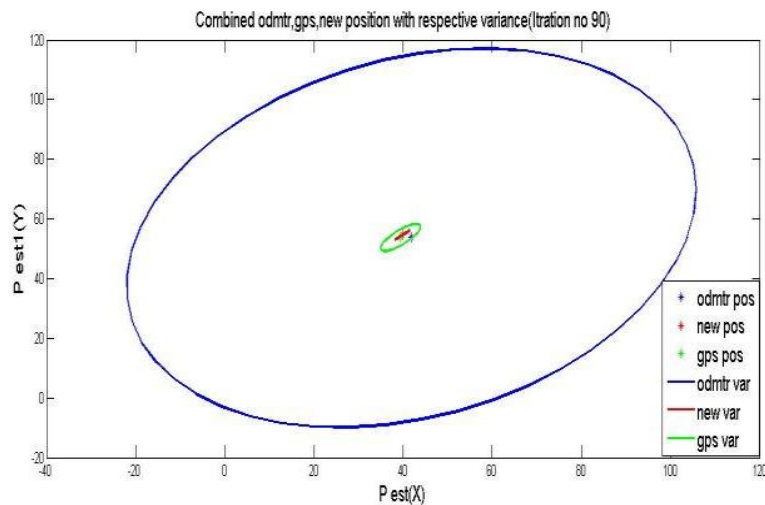

Fig-8,Itration where Odometer variance becomes much greater than GPS and new position variance.

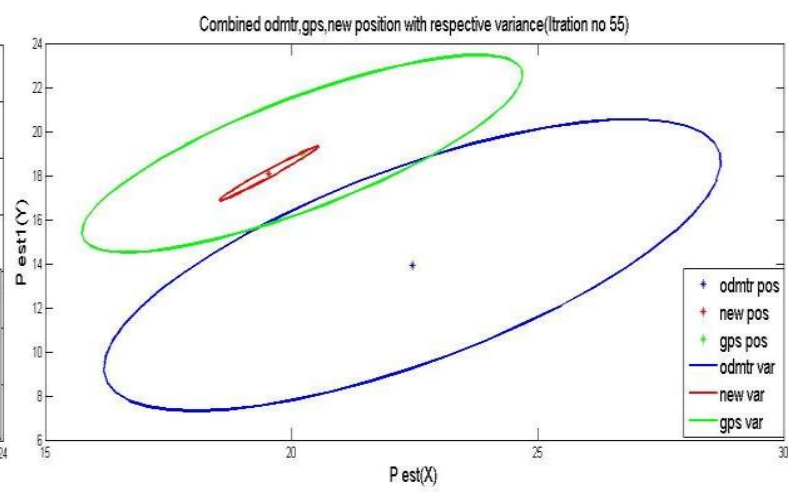

Fig-7,Itration where Odometer variance becomes greater Than GPS variance.

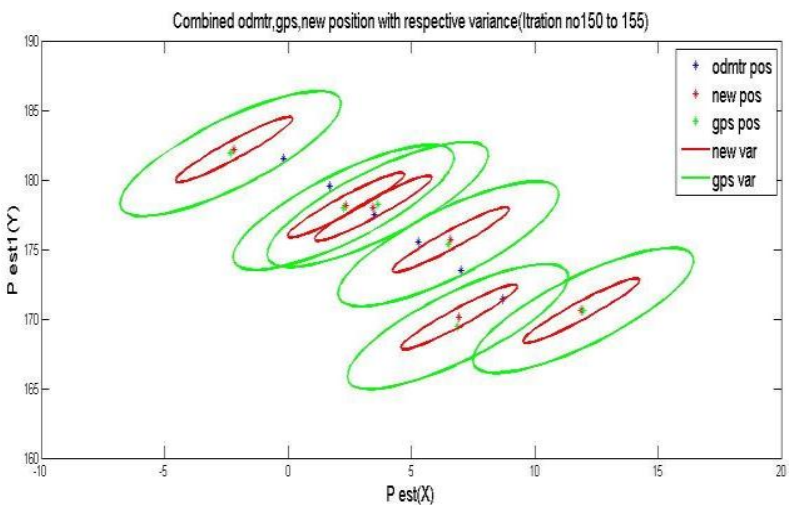

Fig-9,Itration after which new position variance is almost constant $(60 \%$ to70\% of GPS Variance).

\begin{tabular}{|c|c|c|c|c|c|c|}
\hline No of itration & $\begin{array}{c}\text { Odmtr } \\
\text { S.D }(X)\end{array}$ & $\begin{array}{c}\text { Odmtr } \\
\text { S.D }(Y)\end{array}$ & GPS & GPS & n_pos & $n_{-}$pos \\
& 1.47 & 2.40 & 2 & S.D $(Y)$ & S.D $(X)$ & S.D $(Y)$ \\
\hline 50 & 2.02 & 2.92 & 2 & 2 & 0.61 & 0.98 \\
\hline 55 & 9.83 & 6.41 & 2 & 2 & 1.22 & 1.01 \\
\hline 90 & 38.75 & 6.69 & 2 & 2 & $\mathbf{1 . 4 1}$ & $\mathbf{1}$ \\
\hline 150 & 39.30 & 7.08 & 2 & 2 & $\mathbf{1 . 4 1}$ & $\mathbf{1 . 3 3}$ \\
\hline 151 & 39.84 & 7.48 & 2 & 2 & $\mathbf{1 . 4 1}$ & $\mathbf{1 . 3 3}$ \\
\hline 152 & 40.39 & 7.92 & 2 & 2 & $\mathbf{1 . 4 1}$ & $\mathbf{1 . 3 4}$ \\
\hline 153 & 40.93 & 8.37 & 2 & 2 & $\mathbf{1 . 4 1}$ & $\mathbf{1 . 3 4}$ \\
\hline 154 & 58.58 & 46.82 & 2 & 2 & $\mathbf{1 . 4 0}$ & $\mathbf{1 . 4 0}$ \\
\hline 200 & & & & & 21 & \\
\hline
\end{tabular}

Table-1,Comparision between odometer, GPS and new positionvariance( obtained after fusion).All dimensions are in meter.

From simulation we can observed that, initially (fig-5) GPS variance is very high compare to odometer and new variance obtained after fusion. Fig-4shows, due to fusion how a improved position estimation has obtained. After some time Odometer variance will become almost same as GPS variance(fig-6) and slowly become very high compare to GPS and new fused position (fig-7 and fig-8). From the table no-1 It can be observed that after almost 150 iteration new fused position variance ( $\mathrm{X}$ and $\mathrm{Y}$ pos S.D ) is almost constant (fig-9). In Table-1 Odometer, GPS and Fused position variance obtained through simulation has been listed .

\section{Conclusion}

Improved position estimation after odometer and GPS data fusion has been achieved. New position variance is almost $60 \%$ to $70 \%$ of most precise sensor (Odometer before iteration no 50 and GPS after iteration no51).Importance of data fusion for outdoor navigation can be utilise for more precise outdoor/unknown environment missions. Same algorithm can be modified for multi sensor data fusion and in future can be used with Particle filter for more non-linear systems. 


\section{References}

[1] Weiss,J., “Analysis of Upgraded GPS Internal KalmanFilter”. IEEE AES Systems Magagine,Jan-1996.

[2] Barshan,B. And Durrant-Whyte,H., "Inertial Navigation System For Mobile Robots". IEEE Transaction on Mobile Robotics and Automation,Vol.11,No.3,June-1995.

[3] Roland Siegwart and Illah R. NourbakhshIntroduction to Autonomous Mobile Robots" PHI,Eastern Economy Edition.

[4] Oscar E.Ramos Ponce “Autonomous Robots Map-based EKF Localization” Universitat de Girona,April 2010

[5] Bernd Gersdorf and UdoFrese“A Kalman Filter For Odometry using a Wheel Mounted Inertial Sensor". Cyber Physical Systems, German Research Center For Artificial Intelligence,Bremen,Germany.

[6] Laue,T. And Rofer,T.(2008). "SimRobot-Development and Application". In Proceedings of the InternationalConference on Simulation,Modeling and Programming for Autonomous Robots SIMPAR 2008.

[7] Tharun,S.,et al. "Probabilistic Robotics". First Adition, The MIT Press Cambridge,MA,2005. 